\title{
Caracterização de cobertura comestível a base de alginato e óleos essenciais
}

\author{
Characterization of an edible coating based on alginate and essential oils
}

Caracterización de un recubrimiento comestible a base de alginato y aceites esenciales

\section{Resumo}

A utilização de filmes, coberturas ou películas comestíveis aplicadas em produtos tem crescente importância na indústria alimentícia. O alginato é um polissacarídeo muito utilizado e os óleos de alecrim e orégano vêm sendo aplicados como agentes antimicrobianos e antioxidantes. A resistência dessas películas e a transferência de massa através das mesmas são parâmetros importantes e devem ser estudados, dessa forma, esse trabalho teve como objetivo caracterizar cobertura comestível a base de alginato e óleos essências (alecrim e orégano). Nota-se que a adição de óleos não influenciou $(\mathrm{p}<0,05)$ na espessura e solubilidade de água das amostras. A amostra controle (sem óleos essenciais) apresentou maior umidade (aproximadamente $37 \%$ maior) e menor permeabilidade ao vapor de água (65 $\%$ em média). Conclui-se ainda, que a cobertura de alecrim apresentou maior tensão a ruptura e elongação. Deste modo, pode-se concluir que a adição de óleos essências em cobertura comestível a tornou mais resistente se comparado com a amostra sem essa adição, indicando que a mesma apresenta potencial para aplicação em alimentos. Palavras-chave: Embalagem comestível; Óleo essencial de alecrim; Óleo essencial de orégano.

\begin{abstract}
The use of films, coatings or edible films applied to products is of growing importance in the food industry. Alginate is a widely used polysaccharide and rosemary and oregano oils have been applied as antimicrobial and antioxidant agents. The resistance of these films and the mass transfer through them are important parameters and must be studied. Thus, this work aimed to characterize an edible covering based on alginate and essential oils (rosemary and oregano). It can be seen that the addition of oils did not influence $(p<0.05)$ the thickness and water solubility of the samples. The control sample (without essential oils) showed higher humidity (approximately $37 \%$ higher) and lower water vapor permeability (65\% in average). It can also be concluded that the rosemary coating showed higher tensile strength and elongation. Thus, it can be concluded that the addition of essential oils in edible coverings made them more resistant when compared to the sample without this addition, indicating that they have potential for application in food.
\end{abstract}

Keywords: Edible packaging; Rosemary essential oil; Oregano Essential oil.

\section{Resumen}

El uso de películas, recubrimientos o films comestibles aplicados a los productos tiene una importancia creciente en la industria alimentaria. El alginato es un polisacárido muy utilizado y los aceites de romero y orégano se han aplicado como agentes antimicrobianos y antioxidantes. La resistencia de estas películas y la transferencia de masa a través de ellas son parámetros importantes y deben ser estudiados, por lo que este trabajo tuvo como objetivo caracterizar el recubrimiento comestible a base de alginato y aceites esenciales (romero y orégano). Se observa que la adición de aceites no influyó $(\mathrm{p}<0,05)$ en el espesor y la solubilidad en agua de las muestras. La muestra de control (sin aceites esenciales) mostró una mayor humedad (aproximadamente un $37 \%$ más) y una menor permeabilidad al vapor de agua (65\% de media). También se puede concluir que el recubrimiento de romero mostró una mayor resistencia a la tracción y alargamiento. Por lo tanto, se puede concluir que la adición de aceites esenciales en las cubiertas 
comestibles las hizo más resistentes en comparación con la muestra sin esta adición, lo que indica que tienen potencial para su aplicación en los alimentos.

Palabras clave: Envase comestible; Aceite esencial de romero; Aceite esencial de orégano.

\section{Introdução}

O desenvolvimento de formulações de filmes e coberturas comestíveis tem sido crescente nos últimos anos. Este fato sobrevém do aumento da demanda dos consumidores que procuram por produtos com elevada qualidade e vida útil prolongada (Chitarra, 2005). Diferente do que ocorrem com as embalagens usuais, as comestíveis não são planejadas para serem separadas dos materiais embalados, pois são consideradas como parte do produto final (Kester \& Fennema, 1986). Elas possuem a função de inibir ou diminuir a migração de umidade, oxigênio, dióxido de carbono, lipídios, aromas, dentre outros, pois promovem barreiras semipermeáveis. Além do mais, podem carregar ingredientes alimentícios como: antioxidantes, antimicrobianos e flavorizantes, e ainda melhorar a integridade mecânica ou as características de manuseio do alimento no qual ela for aplicada (Krochta \& Mulder-Johnston, 1997).

Dentre os principais polissacarídeos (polímeros naturais) utilizados para formação de embalagens comestíveis, coberturas e filmes destaca-se o amido, alginatos, carragenatos, quitosano e gomas. O alginato é amplamente utilizado na indústria de alimentos, na produção de sorvetes, produtos lácteos, misturas de bolo e algumas de suas aplicações promissores está na utilização em filmes bioativos, elaboração de alimentos reestruturados e cobertura de alimentos. Na área de embalagens, o alginato é um dos compostos naturais mais utilizados devido à suas características como boa formação de filmes, biocompatibilidade, biodegradabilidade e perfil não tóxico (Sirviö et al, 2014; Benavides et al, 2012).

A utilização de óleos essenciais de condimentos, como conservantes, tem sido de grande importância na produção de alimentos (Azevedo et al., 2012). Esses óleos são metabólitos secundários formados por plantas aromáticas e possuem forte odor e propriedades antibacteriana, antifúngica e inseticida. Seus principais compostos são os terpenos e os fenilpropanoides (Oliveira et al., 2013). Os óleos essenciais de orégano e de alecrim são muito usados nas áreas farmacêutica, cosmética e alimentícia (Nascimento, 2007). O óleo de alecrim (Rosmarinus officinalis) tem ação antimicrobiana frente a linhagens bacterianas e também de leveduras (Schelz \& Hohmann, 2006). O óleo essencial de orégano (Origanum vulgareL.) tem apresentado resultados de destaque como agente hábil de inibição de bactérias e fungos contaminantes de alimentos (Souza, 2005). Pouco se sabe sobre a questão estrutural das coberturas, a maioria dos trabalhos da literatura reportam o uso de coberturas e sua relação com a qualidade dos alimentos, assim é de grande importância tal estudo.

A utilização de óleos essenciais com alginato como base de cobertura, foi aplicada em queijo minas frescal em um estudo de Pieretti et al (2019). Nesse estudo foram realizadas análises comparando as amostras de queijo sem cobertura, com cobertura a base de alginato acrescido de cloreto de cálcio, a mesma cobertura com adição óleo de alecrim a 0,1\% e ainda outra cobertura com adição de óleo de orégano a $0,06 \%$. Os resultados encontrados pelos autores em relação à perda de massa foram que a amostra padrão (sem cobertura) obteve perda de $26 \%$, amostra com cobertura sem adição de óleos foi de 16\%, amostra com cobertura e adição de óleo de alecrim $21 \%$ e cobertura com adição de óleo de orégano foi de $20 \%$. Pouco se sabe sobre a questão estrutural das coberturas, a maioria dos trabalhos da literatura reportam o uso de coberturas e sua relação com a qualidade dos alimentos, assim é de grande importância tal estudo.

Então, o objetivo deste trabalho foi investigar a caracterização estrutural de coberturas a base de alginato e óleos essenciais (de alecrim e orégano). 


\section{Metodologia}

\section{Materiais}

Para o desenvolvimento das coberturas foram utilizados óleos essenciais de orégano e alecrim ambas da empresa Ferquimå e alginato em pó (FMC Biopolymer).

\section{Elaboração das Coberturas}

Para o preparo das coberturas foi adicionado o alginato em uma concentração de $2 \%$ em água destilada a $70^{\circ} \mathrm{C}$ por 5 minutos, o mesmo foi agitado constantemente ( $1500 \mathrm{rpm}$ por 6 minutos), resfriado até a temperatura ambiente $\left(25^{\circ} \mathrm{C}\right)$ até sua total solubilização (Groppo et al., 2009). Posteriormente foi acrescentado ou não os óleos estudados neste trabalho (Tabela 1). As alíquotas das soluções filmogênicas foram vertidas em placas com diâmetro de $9 \mathrm{~cm}$ e secas a $24^{\circ} \mathrm{C}$ por 24 horas (Fakhouri, 2007; Pieretti et al., 2019).

Tabela 1. Descrição dos tratamentos.

\begin{tabular}{ll}
\hline Cobertura & Formulação \\
\hline Controle & Cobertura com algintado a $2 \%$. \\
Orégano & Cobertura com alginato a $2 \%$ e óleo essencial de orégano a $0,06 \%$. \\
Alecrim & Cobertura com alginato a $2 \%$ e óleo essencial de alecrim a $0,1 \%$. \\
\hline
\end{tabular}

Fonte: Autores.

\section{Determinação de espessura}

As espessuras das coberturas comestíveis foram determinadas por meio de um micrômetro do estilo do Modelo MDC25M, Mitutoyo, MFG, Japão. As amostras foram cortadas no formato de retângulo de $4 \mathrm{~cm}$ por $2 \mathrm{~cm}$ em decaplicata e medida a espessura em três pontos distintos.

\section{Determinação do teor de umidade}

Determinou-se a umidade de acordo com a proposta oferecida pelas Normas Analíticas do IAL, na qual a secagem foi feita em estufa de $105^{\circ} \mathrm{C}$ durante 24 horas em triplicata (IAL, 1985).

\section{Permeabilidade ao vapor de água}

A permeabilidade ao vapor de água foi determinada gravimetricamente a $25^{\circ} \mathrm{C}$, de acordo com o método ASTM2. Em triplicata, as coberturas foram acondicionadas em dessecador a $25^{\circ} \mathrm{C}$, umidade relativa de $52 \% \pm 2$, por 48 horas. Então, foi montada as cápsulas com as amostras e feita as medições três vezes ao dia durante 4 dias.

\section{Solubilidade em água}

A solubilidade em água das coberturas foi determinada em triplicata, sendo que amostras foram cortadas em quadrados de $2 \mathrm{~cm}$ por $2 \mathrm{~cm}$. A porcentagem inicial da matéria seca de cada amostra foi determinada em estufa a $105^{\circ} \mathrm{C}$ por 24 horas. Após a primeira pesagem, as amostras foram imersas em recipientes contendo $50 \mathrm{~mL}$ de água destilada, e agitadas lenta e periodicamente por 24 horas e a $25^{\circ} \mathrm{C}$. Após este período, as amostras foram removidas e secas ( $105^{\circ} \mathrm{C}$ por 24 horas $)$ e determinada a massa da matéria seca que, a qual será considerada a massa não dissolvida (Fakhouri, 2007). 


\section{Propriedades mecânicas}

A resistência à tração e a porcentagem de elongação na ruptura foram determinadas utilizando o texturômetro, operando de acordo com o método padrão ASTM1 $\left(25^{\circ} \mathrm{C}\right.$, separação inicial das garras $30 \mathrm{~mm}$ e velocidade do probe 100 $\mathrm{mm} /$ minuto). As coberturas foram devidamente cortadas formando corpos de prova de $80 \mathrm{~mm}$ de comprimento e $20 \mathrm{~mm}$ de largura. Desse modo, foram acondicionados por dois dias à temperatura ambiente e umidade relativa de $52 \%$ e então foi feita a medição em decaplicata.

\section{Análise dos dados}

Os dados foram analisados no programa SISVAR 5.3 Por ANOVA e Teste de Tukey $(\mathrm{P}<0,05)($ Ferreira, 2014).

\section{Resultados e Discussão}

\section{Espessura}

A determinação da espessura foi realizada em 3 pontos distintos das amostras e as medidas foram realizadas em decaplicata. Os valores médios encontrados podem ser observados na Tabela 2.

Tabela 2. Valores médios para análise de espessura das amostras de cobertura.

\begin{tabular}{cc}
\hline Formulação & Média das Espessuras (mm) \\
\hline Controle & $0,022^{\mathrm{a}} \pm 0,007$ \\
Orégano & $0,019^{\mathrm{a}} \pm 0,009$ \\
Alecrim & $0,024^{\mathrm{a}} \pm 0,007$ \\
\hline
\end{tabular}

Fonte: Autores.

A adição de óleos essenciais não alterou a espessura das amostras, não sendo observada grande variação nos valores de espessura. Assim, a técnica utilizada foi efetiva, sendo que a solução filmogênica submetida à secagem em estufa foi realizada de forma uniforme. Tal fato provavelmente ocorreu em função da baixa concentração de óleo utilizada.

\section{Umidade}

A amostra controle (apenas alginato a $2 \%$ ) teve maior percentual de umidade $(55,38 \%)$ provavelmente por não possuir óleo essencial em sua composição (Tabela 3). Sabe-se que a atividade antioxidantes dos óleos essenciais em geral é atribuída a constituintes como por exemplo, compostos fenólicos, como os flavonóides, os ácidos fenólicos e os diterpenos fenólicos, sendo que $90 \%$ desta atividade é atribuída aos compostos hidrofóbicos (Justo et al., 2008). Assim, a presença de óleo pode ter influenciado na redução do percentual de umidade, sendo que os óleos são hidrofóbicos e o alginato hidrofílico (Lima et al, 2007).

Tabela 3. Teor de umidade das amostras de cobertura.

\begin{tabular}{cc}
\hline Formulação & Umidade $(\%)$ \\
\hline Controle & $55,38^{\mathrm{a}} \pm 2,71$ \\
Orégano & $33,80^{\mathrm{b}} \pm 1,03$ \\
Alecrim & $35,65^{\mathrm{b}} \pm 1,85$ \\
\hline
\end{tabular}

Fonte: Autores. 


\section{Permeabilidade de vapor de água}

A permeabilidade de vapor de água foi determinada em triplicata e os valores médios encontrados podem ser observados na Tabela 4.

Tabela 4. Valores médios para permeabilidade ao vapor de água das amostras.

\begin{tabular}{cc}
\hline Formulação & $\boldsymbol{K}^{W}\left(\mathbf{x 1 0}^{\mathbf{1 1}}\right)(\mathbf{g} / \mathbf{m}$. Pa.seg$)$ \\
\hline Controle & $9,71^{\mathrm{a}} \pm 0,21$ \\
Orégano & $1,12^{\mathrm{b}} \pm 0,24$ \\
Alecrim & $2,28^{\mathrm{b}} \pm 0,26$ \\
\hline
\end{tabular}

Fonte: Autores.

Assim como na análise de umidade, a presença dos óleos essenciais apresentou influencia, diminuindo a permeabilidade ao vapor de água das amostras. Pode-se ressaltar que este valor está próximo a outro encontrado na literatura, por exemplo, o estudo realizado por Fontes et al (2011) em que analisou-se a permeabilidade do vapor de água em um filme a base de alginato, obtendo um resultado de 15,57 (com espessura de 0,049 mm), assim esse valor é condizente ao encontrado nesse trabalho, que foi de 9,71 (com espessura de 0,022 mm). Levando em consideração que quanto maior a espessura maior é a permeabilidade.

\section{Solubilidade em água}

A solubilidade em água foi determinada e os valores médios encontrados podem ser observados na Tabela 5.

Tabela 5. Valores médios para solubilidade em água das amostras

\begin{tabular}{cc}
\hline Formulação & Solubilidade em água (\%) \\
\hline Controle & $99,98^{\mathrm{a}} \pm 0,01$ \\
Orégano & $99,95^{\mathrm{a}} \pm 0,02$ \\
Alecrim & $99,94^{\mathrm{a}} \pm 0,02$ \\
\hline
\end{tabular}

Fonte: Autores.

Não houve diferença estatísticas entre as amostras de cobertura. Os valores encontrados mostram que as mesmas são bastante solúveis, esse fato é decorrente do alginato ser um composto hidrofílico o que faz com que as coberturas sejam solúveis em água (Alves et al., 2011). Nascimento et al. (2021) avaliando filmes de alginato a $2 \%$ encontraram valores entre 98,91 e $99,15 \%$, corroborando assim com os dados obtidos nesse trabalho em relação a solubilidade.

\section{Propriedades mecânicas}

As propriedades mecânicas, tensão na ruptura e elongação, foram determinadas e os valores encontrados seguem nos histogramas representados nas Figura 1 e Figura 2. 
Figura 1. Valores médios para a tensão na ruptura das amostras.

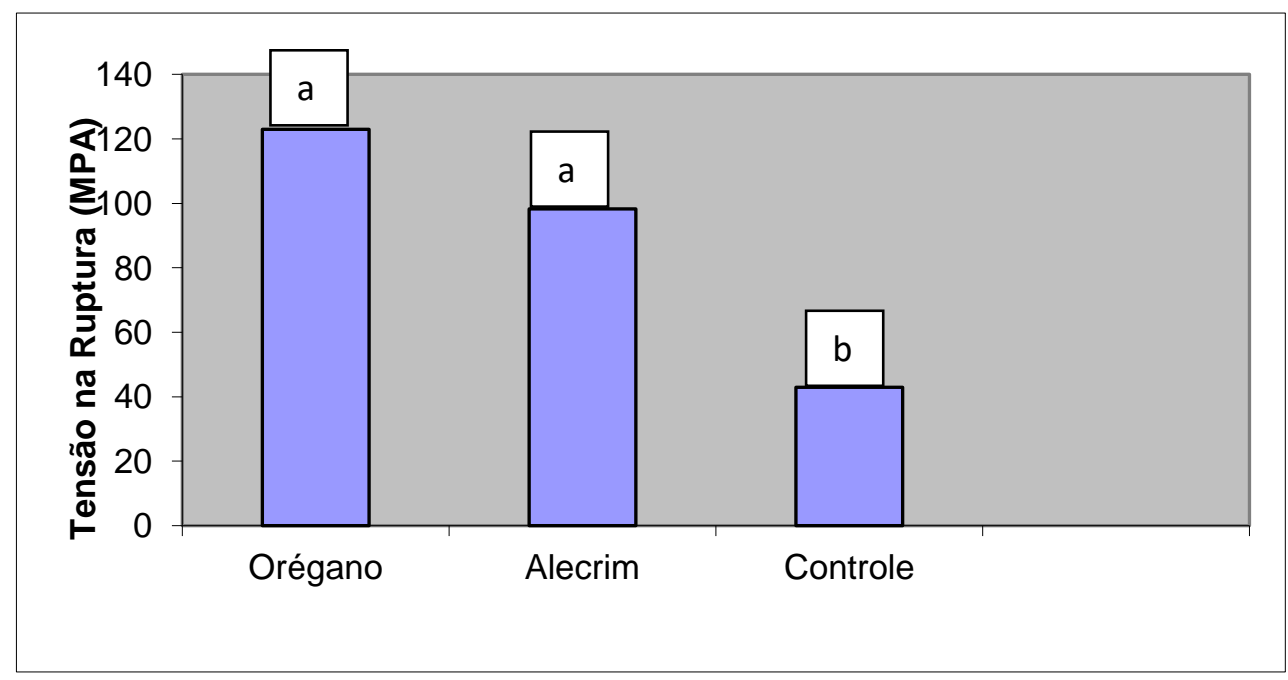

Fonte: Autores.

Figura 2. Valores médios para a elongação na ruptura das amostras.

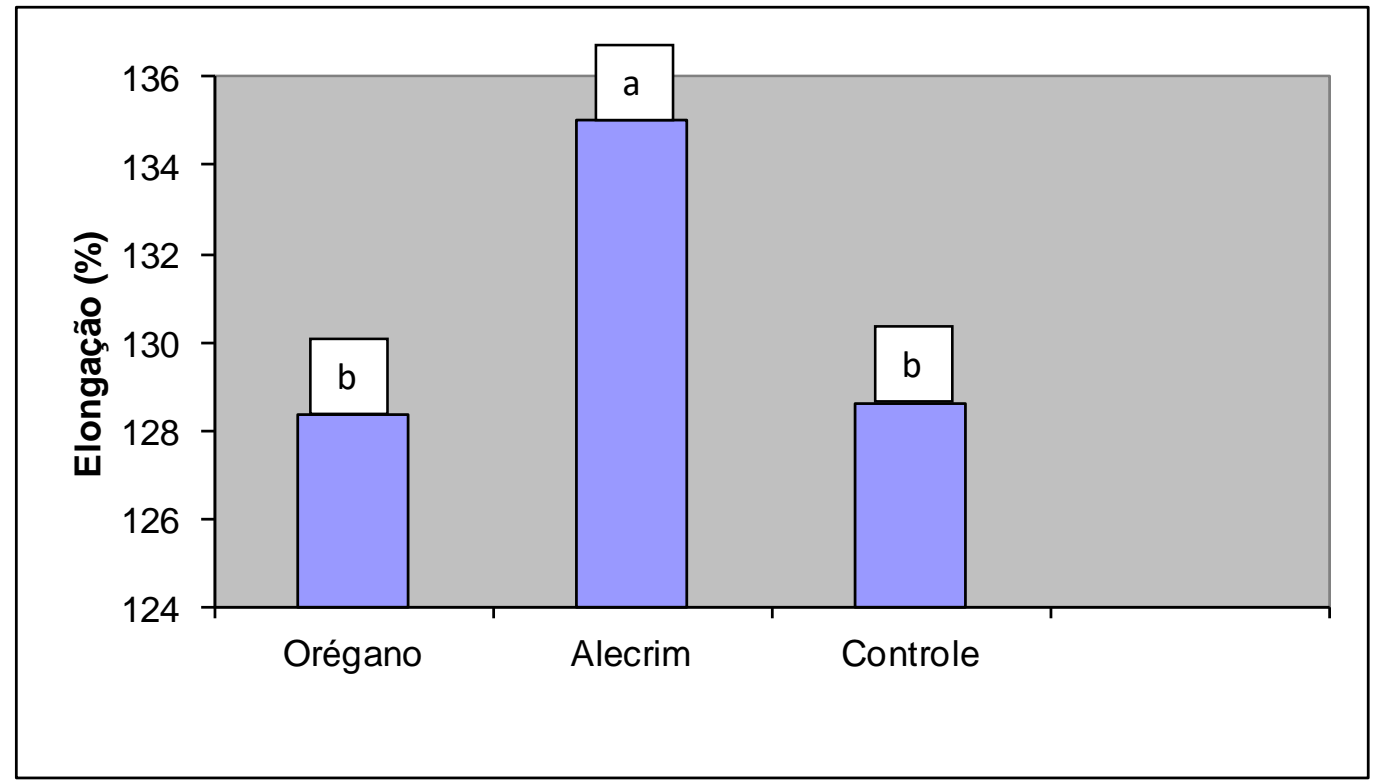

Fonte: Autores.

Os resultados apontam que nas amostras com a adição de óleos essências tem-se maior resistência (120 e 100 MPA) e maior elongação (135 para amostra com óleo essencial de alecrim). Esses números são satisfatórios, pois compreende que a adição de óleo essencial aumenta a resistência da cobertura, não a rompendo com facilidade. Nascimento et al. (2021) avaliando filmes com extrato de barbatimão em forma microencapsulada encontraram valores acima de 70 Mpa, sendo estes maiores que o controle (na forma livre obtiveram valor de $29 \mathrm{Mpa}$ ) para a tensão de ruptura, resistência dos filmes.

Por fim, cita-se que os filmes obtidos neste trabalho se mostraram com boa resistência e boas características, sendo potencialmente indicados para utilização em matrizes alimentícias. Assim, como completo os mesmos foram adicionados em queijo minas frescal e reduziram a perda de massa, melhorando a maciez (textura) e mantendo estáveis a composição físicoquímica, cor e pH. O índice de aceitação sensorial foi alto (IA>75\%), destacando-se a amostra com cobertura adicionada de óleo essencial de orégano, a qual apresentou resultado satisfatório em todos os quesitos e maior índice de aceitação. Assim, de 
forma geral, a adição de cobertura comestível com óleo essencial de orégano e alecrim (nas concentrações estudadas) é uma opção para melhorias nos parâmetros tecnológicos de qualidade (microbiológica, físico-química e sensorial) de queijo minas frescal (Pieretti et al., 2019).

\section{Conclusão}

A utilização de coberturas comestíveis adicionadas de óleos essenciais de orégano e alecrim teve resultados positivos nas condições avaliadas, sendo que a aplicação desses óleos não influenciou a espessura e solubilidade a água das amostras. A amostra controle (sem óleos essenciais) apresentou maior umidade (aproximadamente $37 \%$ maior) e menor permeabilidade ao vapor de água. Conclui-se ainda, que a cobertura de alecrim apresentou maior tensão a ruptura e elongação, por fim, nota-se que a adição de óleos essenciais em cobertura comestível a torna mais resistente se comparado com a amostra sem essa adição.

Ainda, sugere-se como trabalhos futuros o uso de outros óleos essenciais com diferentes concentrações em coberturas comestíveis, e também a aplicação das mesmas em variados tipos de alimentos.

\section{Agradecimentos}

Os autores agradecem a Fundação Araucária, Capes e CNPq.

\section{Referências}

Alves, V. D., Castelló, R, Ferreira, A. R., Costa, N, Coelhoso, I. M. (2011) Barrier properties of carrageenan/pectin biodegradable composite films, Procedia Food Science, 1, 240-245.

$\mathrm{ASTM}_{1}$ (American Society for TestingandMaterials). (1980) Method D 882-83: Standard testmethods for tensilepropertiesofthinplasticsheeting. In: Annual book of ASTM standards. Conshohocken, PA.

$\mathrm{ASTM}_{2}$ (American Society for TestingandMaterials). (1980) Method E 96: Standard testmethods for water vapor transmissionofmaterials. In: Annual book of ASTM standards. Conshohocken, PA.

Azevedo, V. M, Costa, J. M, Domingo, E. C, Carneiro, J. D. S., Pinto, S. M., \& Borges, S. V. (2012) Potencial de aplicação de microcápsulas de óleo essencial de orégano em queijo quark. Inst. Latic. "Cândido Tostes”, 67, 5-12.

Benavides, S., Villalobos-Carvajal, R., \& Reyes, J. E. (2012) Physical, mechanical and antibacterial properties of alginate film: Effect of the crosslinking degree and oregano essential oil concentration. Journal of Food Engineering, 110, 232-239.

Chitarra, F. I. M. (2005) Pós-colheita de frutas e hortaliças: fisiologia e manuseio. (2a ed.), FAEPE: 785 p.

Fakhouri, F. M. (2007) Filmes e coberturas comestíveis compostas à base de amidos nativos. Ciências e Tecnologia de Alimentos, Campinas - SP.

Ferreira, D. F. (2014) Sisvar: a Guide for its Bootstrap procedures in multiple comparisons. Ciênc. Agrotec, 38(2), 109-112.

Fontes, L. C. B., Ramos, K. K., Sivi, T. C., \& Queiroz, F. P. C. (2011) Biodegradable Edible Films from Renewable Sources-potential for their Application in Fried Foods. American journal of food technology, 6, 555-567.

Groppo, V. D. (2009) Effect of calcium chloride and sodium alginate coating in the conservation of 'Pera' orange minimally processed. Food Sciences and Tecnology, 29(1), 107-113.

IAL.(1985) Métodos Químicos e físicos para análise de alimentos. In: IAL.(1985) Normas Analíticas do Instituto Adolfo Lutz. (3a ed.), 533.

Justo, O. R., Moraes, A. M., Barreto, G. P. M., Mercadante, A. Z., \& Rosa, P. T. (2008) Avaliação do potencial antioxidante de extratos ativos de plantas obtidos por extração com fluido supercrítico. Química Nova, 31(7), 1699-1705.

Kester, J. J., \& fennema, O. R. (1986) Edible Films and Coatings: A Review. Food Technology, 40, 47-59.

Krochta, J. M., \& Mulder-Johnston, C. (1997) Edible and biodegradable polymer films: challenges and opportunities. Food Technology, 51, 61-74.

Lima, A. M. F., Andreani, L., \& Soldi, V. (2007) Influência da adição de plastificante e do processo de reticulação na morfologia, absorção de água e propriedades mecânicas de filmes de alginato de sódio. Química Nova, 30(4), 832-837.

Nascimento, P. F. C. (2007) Antimicrobial activity of the essentials oils: a multifactorapproach of the methods. Revista Brasileira Farmacognosia, 17, 108113.

Nascimento, K. M., Cavalheiro, J. B., Netto, A. Á. M., Scapim, M. R. S., \& Bergamasco, R. C. (2021) Properties of alginate films incorporated with free and microencapsulated Stryphnodendron adstringens extract (barbatimão). Food Packaging and Shelf Life, 28, 100637. 
Research, Society and Development, v. 11, n. 2, e52911226145, 2022

(CC BY 4.0) | ISSN 2525-3409 | DOI: http://dx.doi.org/10.33448/rsd-v11i2.26145

Oliveira, M. M. M., Brugnera, D. F., \& Piccoli, R. H. (2013) Biofilmes em indústrias de laticínios: aspectos gerais e uso de óleos essenciais como nova alternativa de controle. Inst. Latic. "Cândido Tostes", 68(390), 65-73.

Pieretti, G., mikcha, J. M. G., Scapim, M. R. S., \& Madrona, G. S. (2019) Effect of an edible alginate coating with essential oil to improve the quality of a Fresh cheese. Acta Scientiarum. Technology (Online), 41, 36402.

Schelz, Z. M. J., \& Hohmann, J. (2006) Antimicrobial and antiplasmid activities of essential oils. Fitoterapia, 77, 279-285.

Sirviö, J. A., Kolehmainen, A., Liimatainen, H., Niinimäki, J., \& Hormi, O. E. O.. (2014) Biocomposite cellulose alginate films: Promising packaging materials. Food Chemistry, 151, 343-351.

Souza, E. L. (2005) Orégano (Origanum Vulgare L., Lamiaceae): Uma especiaria como potencial fonte de compostos antimicrobianos. Revista Higiene Alimentar, 19, 40-45. 